\title{
Proper motions of stars in the region of the Orion Nebula cluster $(\mathrm{C}$ 0532-054)^
}

\author{
K.P. $\operatorname{Tian}^{1,2}$, F. van Leeuwen ${ }^{3}$, J.L. Zhao ${ }^{2,1}$ and C.G. Su${ }^{1}$ \\ 1 Shanghai Observatory, Chinese Academy of Science, Shanghai 200030, China \\ 2 CCAST(World Laboratory) P.O.Box 8730,Beijing 100080, China \\ 3 Royal Greenwich Observatory, Madingley Road, Cambridge CB3 0EZ, UK
}

Received May 26 1994; accepted May 20, 1995

\begin{abstract}
Relative proper motions and membership probabilities for 333 stars within an area of 1.6 by 1.8 centred on the Orion Nebula M 42 are determined using plates taken over a period of 83 years with the double astrograph of Shanghai Observatory (scale of $30^{\prime \prime} / \mathrm{mm}$ ). The plates were measured with the ASTROSCAN automatic platemeasuring machine of Leiden Observatory. The average proper motion accuracy obtained for stars in the photographic magnitude range 7 to 14 is 0.3 mas/yr. Errors are somewhat larger towards fainter and brighter magnitude, but the majority lie well below 1 mas/yr. $64 \%$ of the stars have been measured successfully on at least 13 out of 18 plates. The number of stars with membership probabilities higher than 0.7 is 184 . It is shown by a detailed discussion that the proper motions and membership probabilities of the stars determined in this paper are in good agreement with the results presented recently by other authors. Although there is a clear concentration in the proper motion diagram, both the remaining dispersion of the internal motions and the distribution of "members" as projected on the sky indicate that the stars in this region are not bound as one system, but do have a common origin. A similar conclusion can be drawn from a comparison with spectroscopic and radial velocity data.
\end{abstract}

Key words: open clusters — C 0532-054 — astrometry

\section{Introduction}

Stars in the region of Orion Nebula M 42 play an important role in our understanding of pre-main-sequence stellar evolution. This region has long been a focal point for studies of star formation. It contains most of the signatures of star formation, including an HII region, TTauri stars, high luminosity embedded infrared sources, OB stars and a massive molecular cloud. We will refer to the stars in this region as members of the Orion Nebula cluster (C 0532-054), although it will be shown later that this is not likely to be a dynamically bound stellar system. Proper motion studies have been one of the principal tools used for determination of cluster membership, but in the case of the Orion region, members also stand out through characteristics of their very young age (e.g. TTauri stars).

The Orion Nebula cluster is located $19^{\circ} 4$ below the galactic plane and hence the number of non-clustermember stars in the field is not very large. In addition,

Send offprint requests to: K.P. Tian

${ }^{\star}$ Table 3 is only available in electronic form at the CDS via anonymous ftp 130.79.128.5 the Orion nebulae (M 42 and M 43, NGC 1973, 1975 and 1977) and the associated dust clouds in the area obscure most of the background stars. The majority of the noncluster members should therefore be between the sun and the cluster (distance: $\sim 470 \mathrm{pc}$, van Altena et al. 1988) and should generally have a relatively large proper motion. These circumstances facilitate the cluster membership analysis. The same dust clouds can, however, also complicate the study of the space density distribution in the cluster.

An extensive study of proper motions of stars in the Orion region, covering a $3^{\circ}$ by $3^{\circ}$ area, was made by Parenago (1954). The proper motions from his study were further analyzed by McNamara \& Huels (1983) to derive membership probabilities. More recent proper motion studies are by McNamara (1976), Jones \& Walker (1988), van Altena et al. (1988), and McNamara et al. (1989). These studies are further described in the section on comparisons with the present data. The radial velocities and spectral types of stars in the same region were studied by Johnson (1965) for brighter stars and by Walker (1982) for stars with $11.0 \leq V \leq 14.5$. A photometric survey of the region is provided by Walker (1969). There exist in 
addition several studies on the many variable stars present in this region of the sky.

In this paper we determine the membership of the Orion Nebula cluster using relative proper motions derived from plates taken at Zô-Sè Observatory near Shanghai. The results are compared with the above-mentioned studies to see to what extent very precise differential proper motion studies give the same results and what can be said about internal proper motion dispersions in the cluster.

\section{Plate measurements and reduction of proper motions}

\subsection{Plate material and measurements}

Eighteen plates of the Orion Nebula area were available for the present study. They were taken with the double astrograph at the Zô-Sè (Sheshan) station of Shanghai Observatory. The telescope and the site were described in detail by Chevalier (1905). Zô-Sè is a small hill situated in the alluvial plane of the Chang Chiang (Yangtze) river, some $30 \mathrm{~km}$ east of the centre of Shanghai. The telescope, built by Gaultier in Paris, has an aperture of $40 \mathrm{~cm}$ and a focal length of $6.9 \mathrm{~m}$, giving a plate scale of $30^{\prime \prime} / \mathrm{mm}$. Plates measuring 24 by $30 \mathrm{~cm}$ were used throughout. The firstepoch plates were taken between 1902 and 1923. These plates were used by Chevalier (1930) in an earlier study of the area. The second epoch plates were all taken in 1985. Table 1 provides details for the 18 plates that were used. Altogether, positions and relative proper motions were derived for 333 stars brighter than photographic magnitude 15.0 in an area covering 1.6 by 1.8 , centred on the Trapezium cluster $\left(\alpha_{\mathrm{J} 2000}=5^{\mathrm{h}} 35^{\mathrm{m}} \cdot 5, \quad \delta_{\mathrm{J} 2000}=-5^{\circ} 27 ! 9\right)$. The sample is complete down to $m_{\mathrm{pg}}=14.5$, except for the areas affected by nebulosity.

All plates were measured on the ASTROSCAN, the fully automatic measuring machine at Leiden Observatory (see e.g. van Leeuwen 1983; De Vries 1986). A pixel size of 20 by 20 microns was used throughout. The plates were only scanned for small areas centred on individual stellar images. The size of each area and its a priori position was determined manually on a reference plate. Provisional proper motions were applied to the preparation file to optimize for plates at different epochs. The positions were transformed by the Astroscan software to a priori positions for the plate on the measuring machine. Stability of the positions was checked by a measuring 12 stars before and after the plate measurement.

The centering algorithm used to extract positional information from the digitized images has been described by van Leeuwen (1983). It is based on a simple cone fit (variable slope and width) to the stellar image, thus determining the stellar positions from their slopes. The same technique was employed electronically (but with fixed slope) in the GALAXY automatic measuring machine at the Royal Greenwich Observatory (Murray \& Nicholson 1975). This
Table 1. Plate details

\begin{tabular}{rrrrr}
\hline Plate No. & $\begin{array}{r}\text { Epoch } \\
(1900+)\end{array}$ & $\begin{array}{r}\text { Exp.time } \\
(\mathrm{min})\end{array}$ & $\begin{array}{r}\text { H.A. } \\
(\mathrm{min})\end{array}$ & $\begin{array}{r}\text { No. stars } \\
(\text { measured })\end{array}$ \\
\hline 39 & 02.92 & 65 & - & 291 \\
210 & 08.02 & - & - & 327 \\
296 & 10.12 & 50 & - & 317 \\
332 & 11.07 & 150 & - & 326 \\
365 & 12.04 & 90 & - & 328 \\
397 & 14.09 & 65 & - & 146 \\
413 & 16.00 & 45 & - & 272 \\
414 & 16.07 & 150 & - & 323 \\
514 & 23.02 & 120 & - & 331 \\
85001 & 85.03 & 40 & $50 \mathrm{~W}$ & 321 \\
85002 & 85.03 & 30 & $44 \mathrm{E}$ & 233 \\
85003 & 85.03 & 45 & $6 \mathrm{E}$ & 177 \\
85004 & 85.03 & 20 & $41 \mathrm{~W}$ & 90 \\
85007 & 85.04 & 20 & $13 \mathrm{E}$ & 324 \\
85008 & 85.04 & 50 & $25 \mathrm{~W}$ & 332 \\
85009 & 85.07 & 22 & $48 \mathrm{E}$ & 192 \\
85010 & 85.07 & 16 & $25 \mathrm{E}$ & 189 \\
85011 & 85.07 & 18 & $12 \mathrm{E}$ & 207 \\
\hline
\end{tabular}

mechanism was enhanced by an interactive mode, where the digitized image was displayed on a colour screen and the operator requested for an a priori position and image radius. Thus it became possible to obtain positions of images disturbed by neighbouring images and by réseau lines (on old epoch plates). The centering algorithm was able to remove discrepant data while iterating towards a satisfactory solution. In some areas positional solution accuracies were badly influenced by the brighter parts of nebulae, where the background signal was pushed so high that little of the profile was left in the stellar image. The Trapezium cluster itself could not be measured for that reason, as its stars were barely visible on most plates.

Table 2 compares the observed positional measuring errors with the average formal error obtained from the least-squares fit to the stellar profile. The increase in measuring errors near the centre of the plate is due to the M42 and M43 nebulae.

\subsection{Proper motions}

The methods outlined by van Leeuwen (1994) have been closely followed in the reductions of the measured stellar positions on plates to positions at a reference epoch and in the deriviation of the proper motions. Using the best new epoch plates, a single-epoch reference system was created. Using stars in common between the best old (6) and new (3) plates, the positions on these 9 plates were transformed using only those stars in common as reference points. This defines a preliminary proper motion system in which the proper motions of the selected stars are collectively free of translation, rotation and expansion. The transformations 
Table 2. Observed measuring errors (in $\mu \mathrm{m}$ ) of stellar images in the Orion Nebula region (1.6 by $\left.1^{\circ} .8\right)$ as a function of magnitude and the distance $(r)$ from the field centre, compared with formal errors from the centering algorithm

\begin{tabular}{|c|c|c|c|c|c|c|c|c|c|}
\hline magn. & & $15^{\prime}$ & $15^{\prime}$ & $=30^{\prime}$ & & $\leq 45^{\prime}$ & & $45^{\prime}$ & formal \\
\hline interval & $N$ & $\mathrm{rms}$ & $N$ & $\mathrm{rms}$ & $N$ & $\mathrm{rms}$ & $N$ & $\mathrm{rms}$ & error \\
\hline $6-8$ & 4 & 4.1 & 19 & 2.1 & 31 & 1.4 & 12 & 1.6 & 1.1 \\
\hline $8-12$ & 2 & 3.5 & 32 & 1.7 & 35 & 1.5 & 23 & 1.5 & 0.7 \\
\hline $12-14$ & 7 & 2.9 & 34 & 1.9 & 40 & 1.7 & 28 & 1.7 & 0.9 \\
\hline $14-15$ & 3 & 3.1 & 19 & 2.0 & 26 & 1.9 & 12 & 1.9 & 1.1 \\
\hline
\end{tabular}

were applied to all stars measured. The proper motion for any star with sufficient observations was calculated within this reference frame. Up to this point, only linear plate parameters were used. In the further reductions tilt parameters were added to the transformations. This means that if any systematic differences in tilt between old and new epoch plates were present, then these differences will inevitably reflect in the final proper motions.

Next, using all stars for which a solution was obtained in the preceding step and applying the proper motion corrections to positions in the reference frame, all plates were reduced. Proper motion solutions and positions were obtained for stars with a minimum number of five measurements spread over both the new and old epoch plates. In the solution for each individual star each position was weighted with the inverse standard error of the positional accuracy obtained in the centering algorithm. This means that even for stars measured on all 18 plates, the mean epoch varies slightly.

In these first steps, the transformation coefficients were limited to first-order magnitude terms only. In the next step, using now all stars for which solutions were obtained, second-, and if justifiable, third-order magnitude terms were included in the plate transformations. Finally, the definition of the proper motion system was adjusted. Three iterations were made between the membership determination (described below) and the transformations of the proper motions, using stars with membership probability greater than 0.7 to define the proper motion reference frame. The final transformations made no significant difference to the proper motions. The proper motions of the selected stars show no systematic effects as linear functions of position and up to second-order in magnitude. These are all effects that can not be determined in a differential proper motion study as they enter as degrees of freedom in the plate transformations. The proper motion system is thus in that sense arbitrary, but internally well defined.

Table 3 (here given for only the first ten entries, but available in electronic form at the CDS via anonymous ftp 130.79.128.5) presents the results of the present study for all 333 stars in this proper motion system. A comparison of the proper motions for 45 stars with data available in the
PPM star catalogue Vol. III (Bastian \& Röser 1993) gives the following proper motion for the cluster with respect to the FK5 system: $\mu_{x}=+0.8 \pm 0.5 \mathrm{mas} / \mathrm{yr}, \mu_{y}=-2.0 \pm 0.5$ mas/yr. By adding these numbers to the relative proper motions in Table 3, values closer to absolute proper motions can be obtained. The J2000 positions were obtained by means of a simple transformation of the tangential projections using linear and tilt related coefficients, to preliminary Tycho positions, based on 30 months of data (Høg et al. 1995). They are reliable to at least $0^{\prime \prime} 1$ (the present data were used in the verification of the completeness of the Tycho data). The positional errors indicated in Table 3 refer to the internal accuracies or precisions of the positions, not to their absolute accuracies. However, with the forthcoming publications of the Hipparcos and Tycho astrometric catalogues, and a transformation of the coordinates to these catalogues (taking into account also possible magnitude effects in the proper motions), these accuracies can become absolute accuracies.

Figures 1 and 2 summarize the characteristics of the proper motions and their accuracies. The main influence on the accuracies are the nebulae in the centre of the field. There seems to be no other significant relation between the accuracies of proper motions of stars and their distances from the field centre, which is probably due to the good quality of the optics of the telescope and the reasonable transformation equations that were adopted for proper motion reduction. Results obtained by a very similar instrument, the Leiden Observatory double refractor, which was also built at the end of the 19th century in the Gaultier workshop, and for which the optics were also made by Paul and Prosper Henry in Paris, show similarly good quality results (Vasilevskis et al. 1979; van Leeuwen 1983).

The standard errors of the proper motions for the majority of the 333 stars are well below \pm 0.5 mas/yr. With proper motions of such accuracy one can confidently determine membership and investigate the internal motion dispersion of the cluster. Figure 3 shows the proper motion vector-point diagram for all the stars measured. It is noted from this diagram (as indicated) that the non-cluster members with Parenago numbers 1052 and 1058 are probably a physical pair, with proper motion 
Table 3. (Extract only, full table available in electronic form at the CDS) Results of the proper motion study (mean epoch relative to $\left.1950.0, \alpha^{*}=\alpha \cos \delta, \sigma_{\alpha}:{ }^{\text {s.001, }} \sigma_{\delta}:{ }^{\prime \prime} 01\right)$

\begin{tabular}{|c|c|c|c|c|c|c|c|c|c|c|c|c|c|c|c|c|c|}
\hline \multirow{2}{*}{$\begin{array}{l}\text { Id } \\
1\end{array}$} & \multirow{2}{*}{$\begin{array}{r}\begin{array}{r}\text { Pare- } \\
\text { nago }\end{array} \\
909\end{array}$} & \multirow{2}{*}{$\begin{array}{c}\text { Phot } \\
\text { magn }\end{array}$} & \multicolumn{3}{|c|}{$\begin{array}{l}\text { RA (J2000) } \\
\text { epoch } 1950\end{array}$} & \multirow{2}{*}{$\begin{array}{r}\sigma_{\alpha} \\
2\end{array}$} & \multicolumn{3}{|c|}{$\begin{array}{c}\operatorname{Dec}(\mathrm{J} 2000) \\
\text { epoch } 1950\end{array}$} & \multirow{2}{*}{$\begin{array}{r}\sigma_{\delta} \\
2\end{array}$} & \multicolumn{2}{|c|}{$\begin{array}{c}\mu_{\alpha} * \quad \sigma \\
\text { mas/yr }\end{array}$} & \multicolumn{2}{|c|}{$\begin{array}{l}\mu_{\delta} \sigma \\
\mathrm{mas} / \mathrm{yr}\end{array}$} & \multirow{2}{*}{$\begin{array}{r}N_{\mathrm{Pl}} \\
\\
9\end{array}$} & \multirow{2}{*}{$\begin{array}{r}\begin{array}{r}\text { mean } \\
\text { epoch }\end{array} \\
5.5\end{array}$} & \multirow{2}{*}{$\begin{array}{r}\text { Prb } \\
\% \\
98\end{array}$} \\
\hline & & & $5^{\mathrm{h}}$ & $32^{\mathrm{m}}$ & $25^{\mathrm{s}} .667$ & & $-4^{\circ}$ & $47^{\prime}$ & $46^{\prime \prime} 64$ & & 0.1 & 0.9 & 0.3 & 0.7 & & & \\
\hline 2 & 919 & 14.24 & 5 & 32 & 27.167 & 1 & -5 & 03 & 34.58 & 3 & 6.2 & 0.4 & -18.8 & 0.8 & 9 & 3.3 & 0 \\
\hline 3 & 925 & 14.17 & 5 & 32 & 28.317 & 2 & -5 & 03 & 06.82 & 2 & 3.2 & 0.7 & -2.6 & 0.6 & 9 & 8.1 & 8 \\
\hline 4 & 943 & 13.90 & 5 & 32 & 33.766 & 1 & -4 & 56 & 27.88 & 2 & -9.7 & 0.4 & -1.7 & 0.5 & 12 & -3.2 & 0 \\
\hline 5 & 962 & 13.14 & 5 & 32 & 35.578 & 1 & -6 & 01 & 39.48 & 3 & -0.4 & 0.6 & -0.8 & 0.7 & 13 & -17.1 & 98 \\
\hline 6 & 953 & 14.20 & 5 & 32 & 35.666 & 2 & -4 & 59 & 49.77 & 4 & 3.4 & 0.7 & -27.3 & 1.2 & 11 & -0.4 & 0 \\
\hline 7 & 971 & 10.37 & 5 & 32 & 38.105 & 1 & -5 & 59 & 41.61 & 1 & -3.1 & 0.5 & -42.1 & 0.4 & 17 & 4.9 & 0 \\
\hline 8 & 967 & 11.55 & 5 & 32 & 38.454 & 1 & -5 & 31 & 17.56 & 1 & -2.9 & 0.3 & -5.7 & 0.3 & 16 & 3.7 & 0 \\
\hline 9 & 974 & 14.40 & 5 & 32 & 41.197 & 1 & -5 & 20 & 43.91 & 2 & 2.3 & 0.6 & -13.3 & 0.7 & 10 & 1.2 & 0 \\
\hline 10 & 972 & 12.47 & 5 & 32 & 41.184 & 1 & -5 & 04 & 24.08 & 1 & -4.2 & 0.4 & -4.0 & 0.4 & 17 & 2.5 & 0 \\
\hline
\end{tabular}
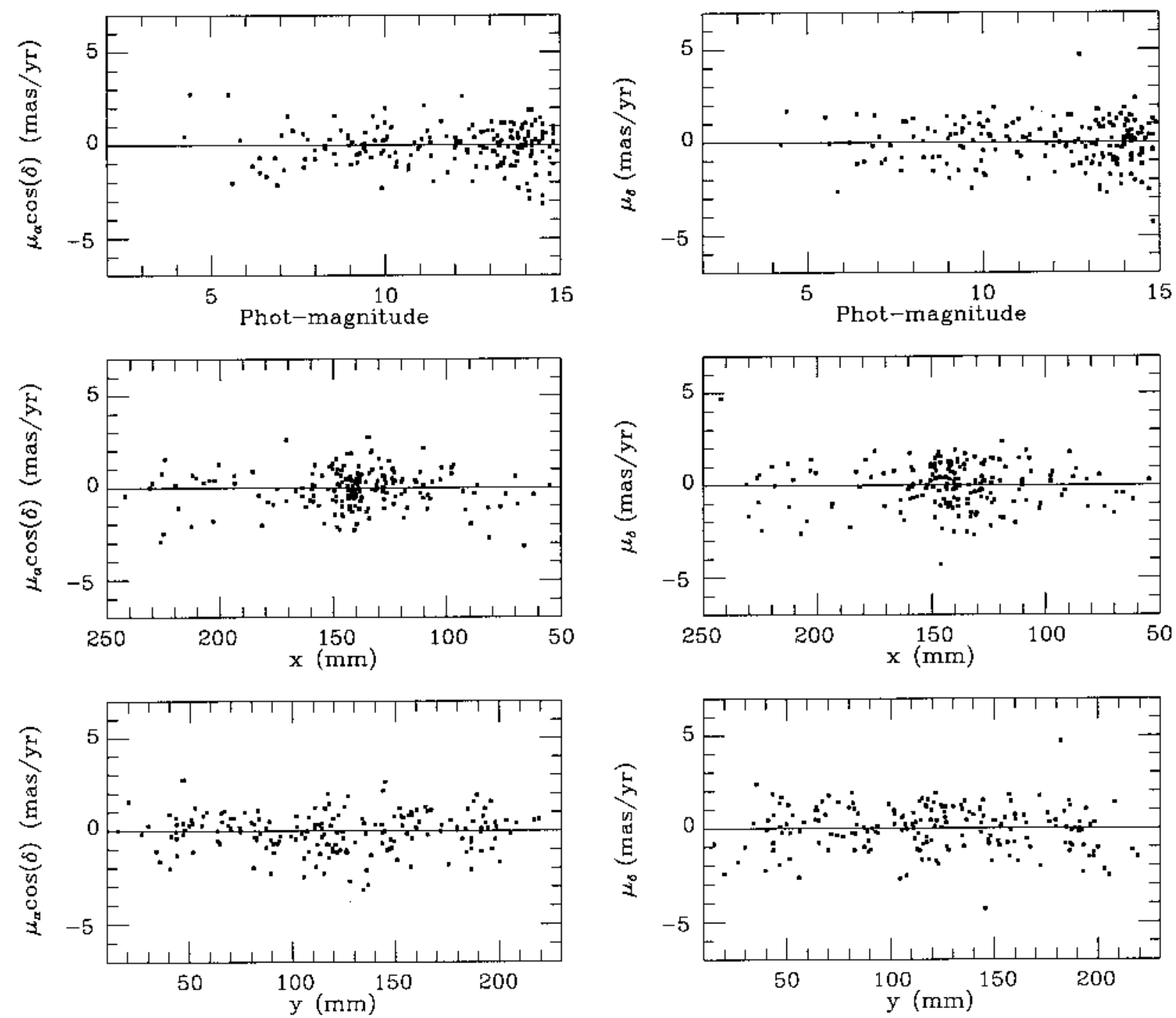

Fig. 1. The proper motions of cluster members as function of position on the plate (centre at $x=140 \mathrm{~mm}, y=120 \mathrm{~mm}$ ) and as function of magnitude 

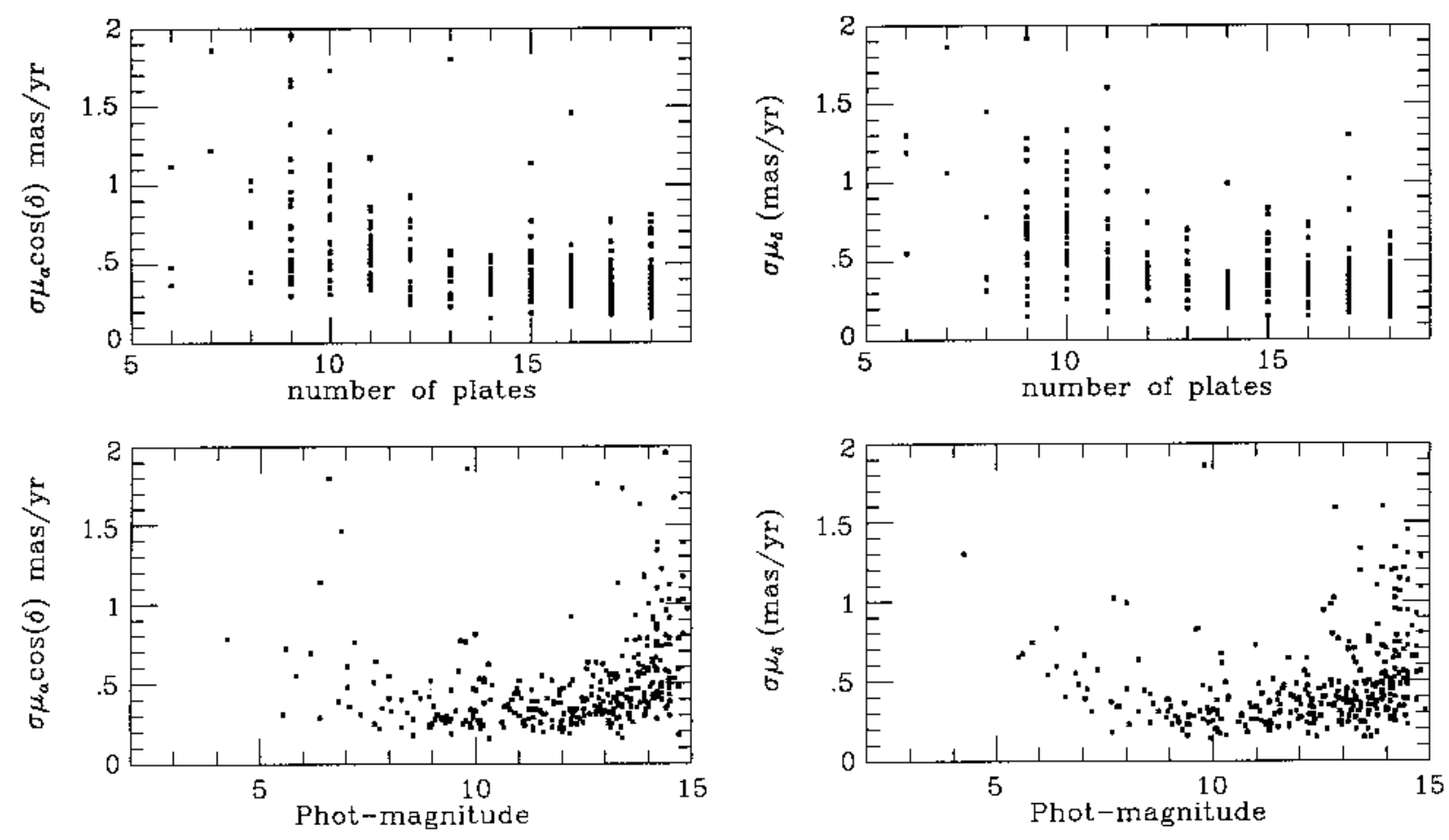

Fig. 2. The proper motion standard errors of all stars measured as a function of magnitude and number of plates used

differences of less than 1 mas/yr, separated in position by $25^{\prime \prime}$ and of nearly the same magnitude and $B-V$ colour, although Walker (1969) shows a rather large difference in the $U-B$ colour index.

\section{Membership determination}

The choice of a model to calculate membership probabilities of stars in a cluster region is important. In consideration of stellar proper motions with obviously different observed errors, the approach suggested by Zhao \& He (1990), which was developed on the basis of Sanders' method (Sanders 1971), is the most appropriate to determine the membership probabilities in the present study. In this model one assumes that the field and cluster proper motion distributions can be represented by two overlapping gaussian distributions. The proper motion dispersion in the $x$ and $y$ directions are assumed equal for cluster stars but not for field stars. Observed errors of individual stars have been taken into account. The distribution parameters and the corresponding uncertainties for the Orion Nebula cluster obtained using this approach are given in Table 4.

The membership probability histogram (Fig. 4) shows a very clear separation of cluster members and field stars. This is partly due to the high accuracies of the stellar proper motions we obtained and partly to the presence of heavy obscuration of background stars by the Orion cloud. The difficult problem of eliminating background
Table 4. Distribution parameters for the Orion Nebula cluster. $\left(N_{\mathrm{c}} \rightarrow\right.$ number of cluster stars; $N_{\mathrm{f}} \rightarrow$ number of field stars; $\mu_{x \mathrm{c}}, \mu_{y \mathrm{c}} \rightarrow$ the mean cluster proper motions in $x$ and $y$ (mas $/ \mathrm{yr}) ; \mu_{x \mathrm{f}}, \mu_{y \mathrm{f}} \rightarrow$ the mean field proper motions in $x$ and $y$ (mas/yr); $\sigma_{\mathrm{c}} \rightarrow$ intrinsic dispersion of cluster proper motions $(\mathrm{mas} / \mathrm{yr}) ; \sigma_{x \mathrm{f}}, \sigma_{y \mathrm{f}} \rightarrow$ intrinsic dispersions of field proper motions (mas/yr); $\theta \rightarrow$ orientation angle of the minor axis of the elliptical field star proper motion distribution)

\begin{tabular}{lrll}
\hline$N_{\mathrm{c}}$ & 181 & \pm & 3 \\
\hline$N_{\mathrm{f}}$ & 152 & \pm & 3 \\
\hline$\mu_{x \mathrm{c}}$ & -0.05 & \pm & 0.03 \\
$\mu_{y \mathrm{c}}$ & 0.06 & \pm & 0.03 \\
$\mu_{x \mathrm{f}}$ & -3.27 & \pm & 0.26 \\
$\mu_{y \mathrm{f}}$ & -0.75 & \pm & 0.19 \\
\hline$\sigma_{x \mathrm{f}}$ & 8.65 & \pm & 0.19 \\
$\sigma_{y \mathrm{f}}$ & 6.40 & \pm & 0.15 \\
$\sigma_{\mathrm{c}}$ & 0.95 & \pm & 0.04 \\
\hline$\theta$ & $78^{\circ}$ & &
\end{tabular}

stars which (almost) share the cluster motion is thus largely avoided. As the Orion Nebula cluster is relatively close to the sun $(\sim 470 \mathrm{pc})$, stars between the cluster and the sun generally show large proper motions, clearly separating them from those of member stars. We find the 


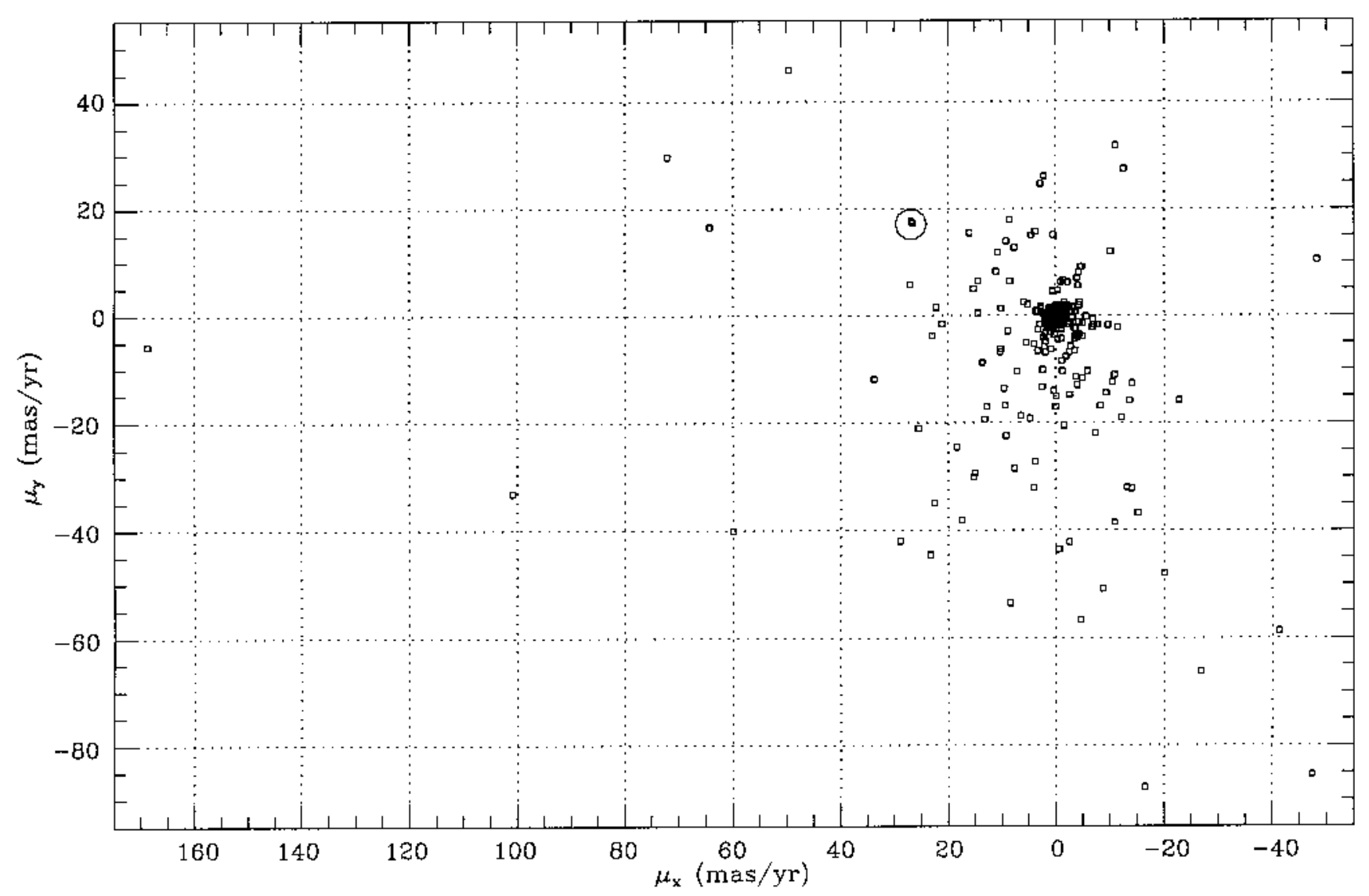

Fig. 3. Proper motions as measured for an area of 1.6 by 1.8 centred on the Orion Nebula M 42 . The proper motions of the probable double star Parenago $1052+1058$ are indicated by the circle

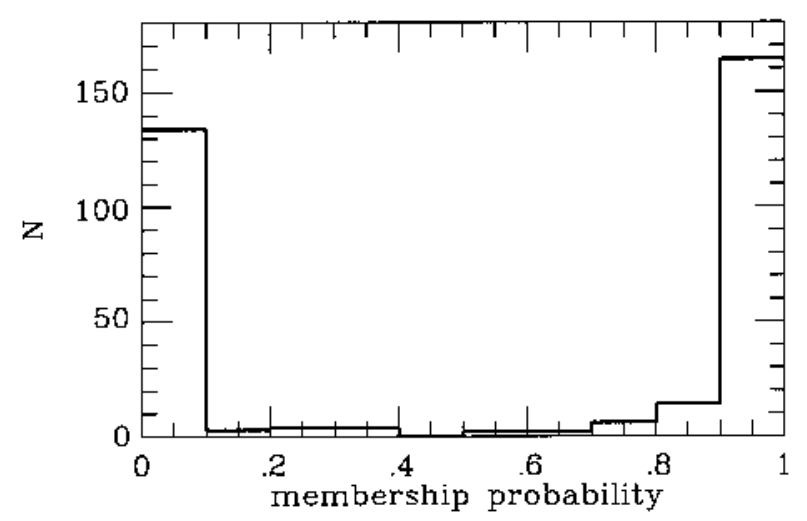

Fig. 4. The histogram of membership probabilities in the Orion cluster

number of stars with membership probabilities higher than 0.7 to be $184(55 \%)$. The total integrated membership probability, i.e. the total number of measured stars likely to be members, is $181(54 \%)$.

\section{Comparisons with other investigations}

The Orion Nebula cluster, which is the nearest one among the very young clusters, provides one of the best opportunities for the study of pre-main sequence evolution of stars and, therefore, there have been many astrometric investigations of the cluster. The aim of these studies generally was the separation of members from field stars. The most notable among them is the classic study by Parenago (1954) of all stars brighter than mag. 17.2 , lying within a 3.0 by 3.0 area round the cluster centre. No separation between members and nonmembers was made by Parenago, but his study did show the widespread variability among stars in this region, which is of a kind associated with very young stars. The proper motions of the Parenago study were re-analysed by McNamara \& Huels (1983) to determine membership probabilities. Strand (1958) measured proper motions in the central region of the Orion Nebula cluster using the Yerkes $100 \mathrm{~cm}$ refractor. McNamara (1976) (MN76), using Harvard and Lick plates with epoch differences of over $80 \mathrm{yr}$, obtained new relative proper motions for some 70 stars over a $65^{\prime}$ by $65^{\prime}$ area centred on the Trapezium cluster. His study also includes a recalibrated 
presentation of the data obtained by Strand (1958), bringing the total number of stars in his paper to around 200. More recently, the cluster was the object of studies by Jones \& Walker (1988) (JW88), van Altena et al. (1988) VA88, and McNamara et al. (1989) (MN89). Details of these investigations, including ours, are listed in Table 5.

In comparing the results of different proper motion studies, two major factors contributing to their determination are revealed. The first is the reference frame within which the proper motions are defined. Simple transformations can produce the same proper motions in different reference frames. The proper motion reference frame can in some cases also contain a component resulting from a change in the average tilt angle between new and old epoch exposures. The second factor is more serious and concerns distortions. These can result from two major causes: plates taken at two epochs separated by a long gap (current study, MN89) during which time the telescope optics may have undergone some (unintended) changes; or the old and new epoch plates from different telescopes in which the field studied is not covered in the same way by plates from these different telescopes (VA88, MN76). A further complication in the study of the relations between proper motion results, comes from underestimating the errors which sometimes gives the impression that the data from different studies ought to agree better than it does.

\subsection{The study by van Altena et al. (1988) (VA88)}

The best way to start a comparison is to use the most rigorous studies available: the study by van Altena et al. (1988) (VA88) and the present one, which have 62 stars in common. Figure 5 shows some of the problems encountered in the comparison with the data presented by VA88. In order to fit the present data with those presented by VA88 it was necessary to include (possibly tilt-related) non-linear coefficients, and to remove two stars on the edge of the common field (which equals VA88's field). These stars are Parenago 1158 and 1212, which both show large (several mas/yr) differences with respect to our study. Also, stars were removed from the present study that due to the closeness of the nebulosity had rather badly determined proper motions. It appears that for those stars the proper motion fitting process had been too generous in removing suspect data points, leaving a faulty proper motion with an underestimated error. Fifty-five stars were left for the transformation, which used six parameters per coordinate: zero shift, $x$-position (in $\mathrm{cm}$ ), $y$-position (in $\mathrm{cm}$ ), tilt terms (not linked) and magnitude. Magnitude squared terms were found not to be significant. Positions, in $\mathrm{cm}$, were taken from the Shanghai plates, $30^{\prime \prime} / \mathrm{mm}$; the proper motions were expressed in mas/yr. Table 6 shows the coefficients found. Figure 6 shows the final comparison between the proper motions of VA88 (after applying the transformation) and the present study. The membership segregation for the 55 stars used in the transformation is identical between the two studies.

Following the results shown above, we checked the current proper motion data for the presence of tilt-related effects, but these were barely significant and amounted to 10 percent of what is given in Table 6 . Applying these tilt corrections appeared to weaken the membership segregation. It was thus decided not to apply a tilt correction to the proper motions of the present study. It should also be emphasized that tilt coefficients as presented in Table 6 extrapolate to errors of 4 to 6 mas/yr on the edge of the Shanghai field, and there is no indication of such an effect in the proper motions of the present study (see also below, the comparison with McNamara et al. 1989). It looks, therefore, more plausible that this effect originates largely from VA88.

\subsection{The study by McNamara (1976) (MN76)}

This study by McNamara is based on 4 plate pairs, where the old plates were taken with the Harvard Boyden $33 \mathrm{~cm}$ refractor $\left(43^{\prime \prime} / \mathrm{mm}\right)$ and the new plates with the Lick $50 \mathrm{~cm}$ Carnegie Astrograph $\left(55^{\prime \prime} / \mathrm{mm}\right)$. Table III of MN76 contains data from these plates, as well as data obtained by Strand (1958) using the Yerkes $100 \mathrm{~cm}$ refractor. In our comparison, 100 stars were used, 67 from the HarvardLick study, and 33 from the Yerkes study. The author assigned standard errors to the proper motions of 0.7 and $1.4 \mathrm{mas} / \mathrm{yr}$ respectively. Figure 7 shows the differences in proper motions between MN76 before and after transformation. In the comparison it appeared that there is a scaling difference between the current proper motions and those presented by MN76. Note that the current proper motions were scaled along with the transformation to absolute coordinates described above, and that therefore as expected, no scaling differences were found in the comparison with the PPM data. Solving for this scaling factor gave a consistent result between the $x$ and $y$ proper motions: in both cases the proper motions in MN76 were approximately $5 \%$ too small. Table 7 and Fig. 6 summarize the transformation and the comparison after application of the transformation. Given the result of the comparison with VA88, the unit weight of standard error of 1.46 results either from a distortion between MN76 and the current data, or from underestimated errors in MN76.

\subsection{The study by Jones \& Walker (1988) (JW88)}

JW88 analyzed proper motions of faint stars in a region measuring $20^{\prime}$ by $30^{\prime}$, centred on the Trapezium cluster. They used 47 plates taken at the prime focus of the Lick Shane $3 \mathrm{~m}$ reflector $\left(13^{\prime \prime} / \mathrm{mm}\right.$, Herbig 1974) in 1960, 1961, 1981 and 1983 . We selected only stars with Parenago numbers (251 stars). The majority of these stars have errors of the proper motions between 0.2 to $0.7 \mathrm{mas} / \mathrm{yr}$, and thus in the same range as the present study. Figure 8 shows the 
Table 5. Comparison with other investigations

\begin{tabular}{|c|c|c|c|c|c|c|c|}
\hline Investigator & $\begin{array}{c}\text { Parenago } \\
\text { (1954) }\end{array}$ & $\begin{array}{l}\text { Strand } \\
\text { (1958) }\end{array}$ & $\begin{array}{c}\text { McNamara } \\
\text { (1976) }\end{array}$ & $\begin{array}{c}\text { Jones \& } \\
\text { Walker } \\
(1988)\end{array}$ & $\begin{array}{c}\text { van Altena } \\
\text { et al. } \\
(1988)\end{array}$ & $\begin{array}{c}\text { McNamara } \\
\text { et al. } \\
(1989)\end{array}$ & $\begin{array}{l}\text { Present } \\
\text { study }\end{array}$ \\
\hline $\begin{array}{l}\text { Scale of plate } \\
\qquad(" / \mathrm{mm})\end{array}$ & $\begin{array}{l}60 \\
32\end{array}$ & 10.65 & $\begin{array}{l}43 \\
55\end{array}$ & 13 & $\begin{array}{l}24.5 \\
14.6 \\
10.6\end{array}$ & 97.9 & 30 \\
\hline $\begin{array}{l}\text { Average epoch } \\
\text { difference }\end{array}$ & $\sim 48$ & $\sim 40$ & $\sim 73$ & $\sim 20$ & $\sim 77$ & $\sim 80$ & $\sim 70$ \\
\hline Area & $3^{\circ}$ by $3^{\circ}$ & $30^{\prime}$ by $30^{\prime}$ & $65^{\prime}$ by $65^{\prime}$ & $20^{\prime}$ by $30^{\prime}$ & $60^{\prime}$ by $40^{\prime}$ & 2.6 by 2.6 & 1.6 by 1.8 \\
\hline $\begin{array}{l}\text { Limiting } \\
\text { magnitude }\end{array}$ & 17.2 & 14.5 & 14 & $\begin{array}{c}\sim 16.0 \\
(\text { Imag. })\end{array}$ & 12.5 & 14.0 & 15.0 \\
\hline No. of plates & $1-40$ & 19 & 8 & 47 & 138 & 96 & 18 \\
\hline No. of stars & 2982 & 224 & 222 & 997 & 73 & 630 & 333 \\
\hline $\begin{array}{l}\text { Mean pm accuracy } \\
\text { for all stars (mas/yr) }\end{array}$ & - & - & 0.70 & 0.80 & 0.19 & 0.69 & 0.54 \\
\hline No. with prob. $\geq 0.7$ & - & - & 146 & 883 & 49 & 220 & 184 \\
\hline $\begin{array}{l}\text { No. of stars common } \\
\text { with present paper }\end{array}$ & - & - & 121 & 61 & 62 & 241 & \\
\hline \multicolumn{3}{|c|}{ as above, for prob. $\geq 0.7$} & 72 & 40 & 36 & 84 & \\
\hline \multicolumn{3}{|c|}{ as above, for prob. $\leq 0.3$} & 32 & 10 & 23 & 107 & \\
\hline
\end{tabular}

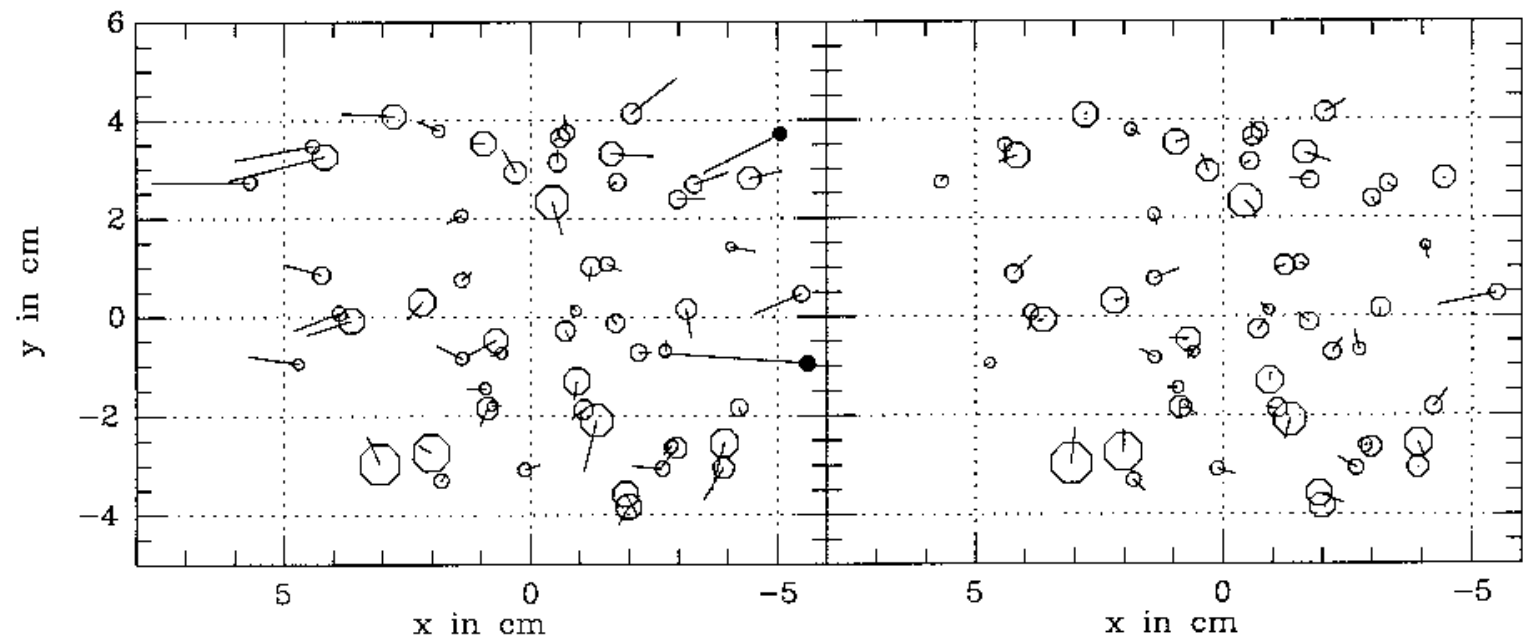

Fig. 5. Vectors on an $x-y$ chart of the field, representing the differences between proper motions presented by VA 88 and the present paper; (left) before application of the transformation, and (right) after. Sizes of the circles refer to the magnitudes of the stars. A vector size corresponding to $1 \mathrm{~cm}$ in the position scale indicates a difference of 2.0 mas/yr. The solid points show the two stars rejected in the transformation 

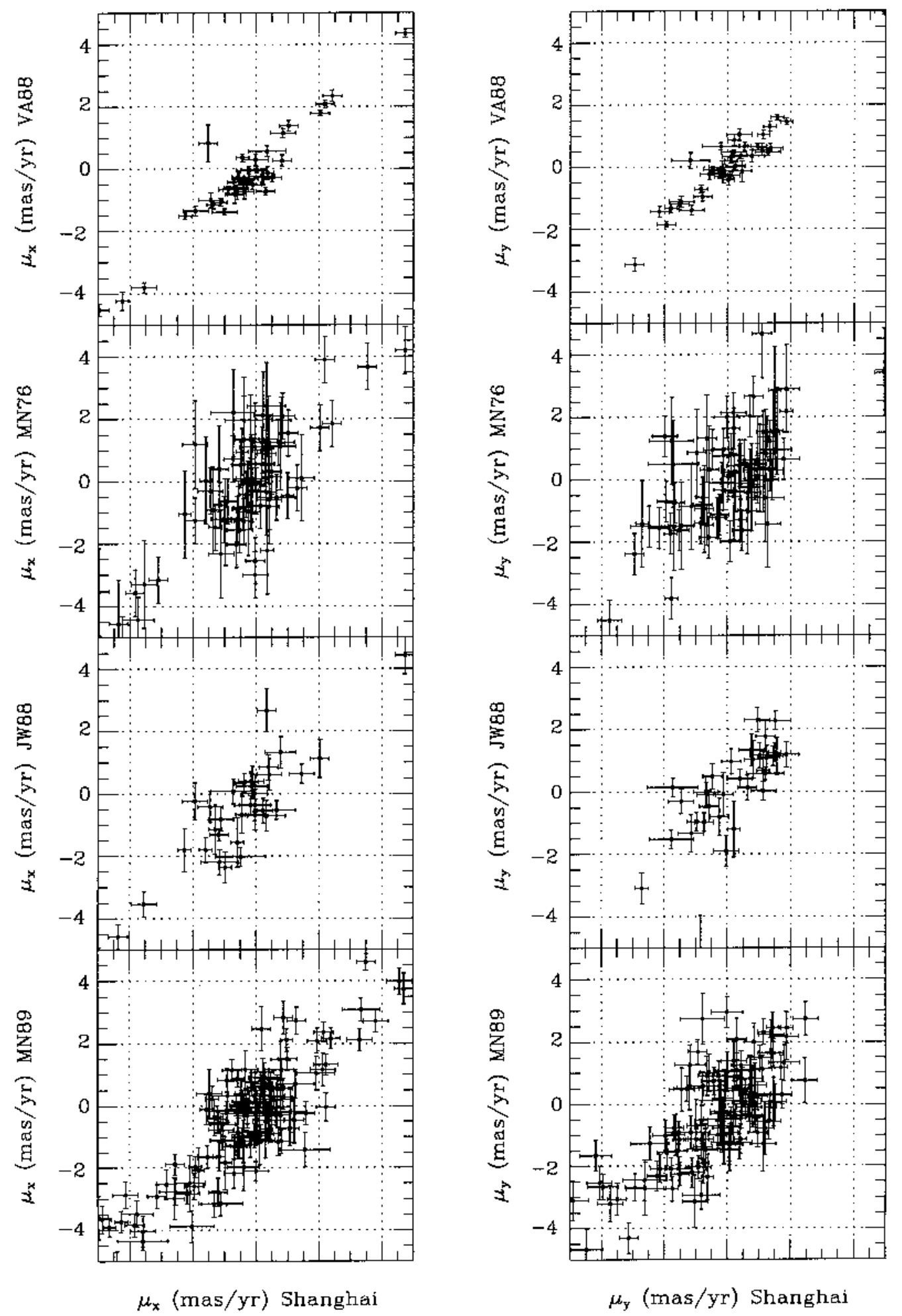

Fig. 6. Comparisons of cluster member proper motions with the present paper. From top to bottom: van Altena et al. (1988), McNamara (1976), Jones \& Walker (1988) and McNamara et al. (1989) 


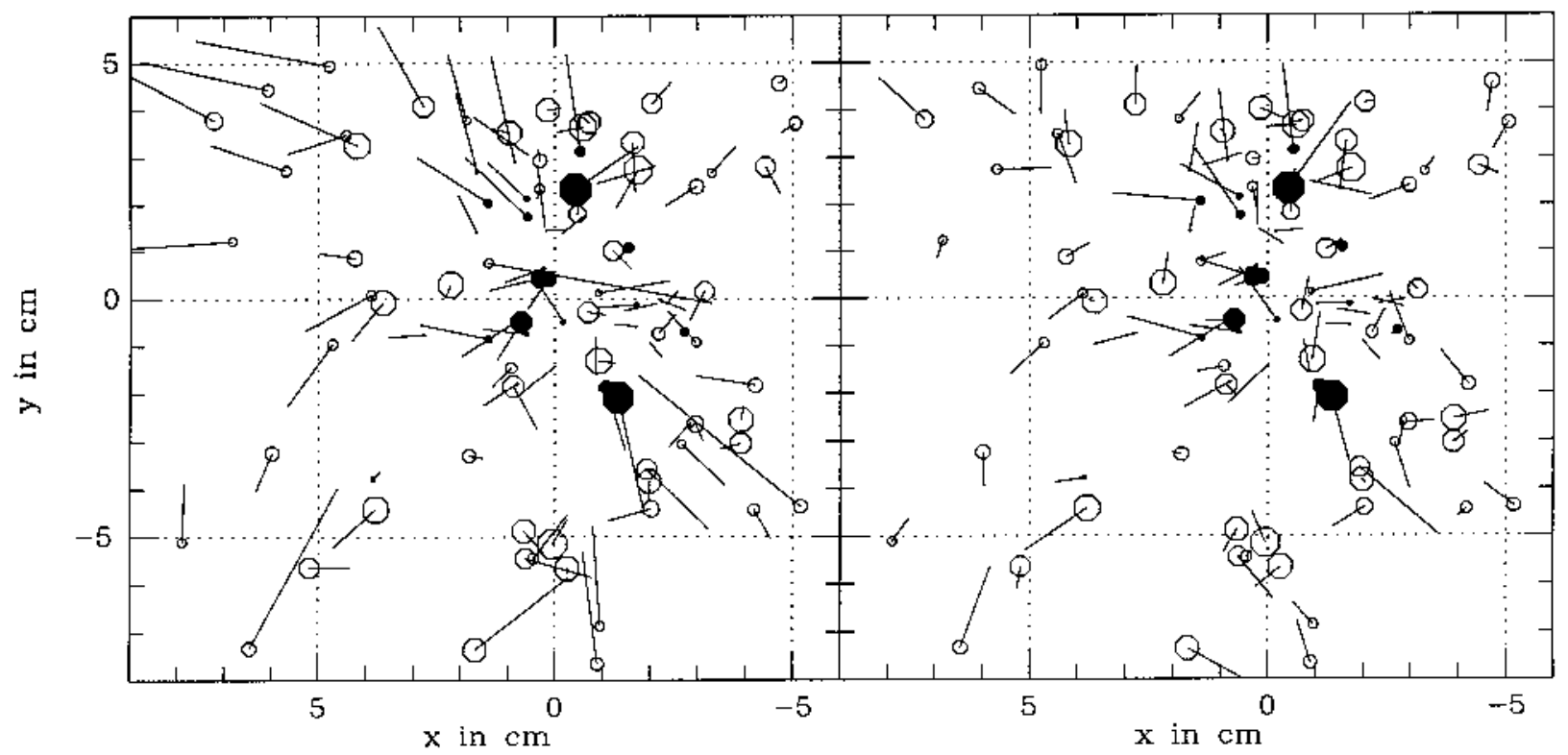

Fig. 7. Vectors on an $x-y$ chart of the field, representing the differences between proper motions presented by MN76 and the present paper; (left) before application of the transformation, and (right) after. Sizes of the circles refer to the magnitudes of the stars. A vector size corresponding to $1 \mathrm{~cm}$ in the position scale indicates a difference of $2.0 \mathrm{mas} / \mathrm{yr}$. The solid points refer to proper motions from Strand (1958)

Table 6. Transformation from VA88 to present data

\begin{tabular}{lrr|lrr}
\hline & $x$-solution & & \multicolumn{3}{c}{$y$-solution } \\
\hline Term & coeff. & $\sigma$ & Term & coeff. & $\sigma$ \\
\hline offset & -0.15 & 0.09 & offset & 0.65 & 0.10 \\
$x(\mathrm{~cm})$ & -0.250 & 0.026 & $x(\mathrm{~cm})$ & -0.070 & 0.025 \\
$y(\mathrm{~cm})$ & 0.008 & 0.028 & $y(\mathrm{~cm})$ & -0.031 & 0.029 \\
$x * x$ & -0.038 & 0.010 & $x * y$ & 0.061 & 0.011 \\
$x * y$ & -0.091 & 0.012 & $y * y$ & -0.061 & 0.012 \\
mag-10 & 0.048 & 0.042 & mag-10 & -0.243 & 0.045 \\
\hline \multicolumn{2}{l}{ Reference stars : } & 55 & unit weight st.dev.: & 1.36 \\
\hline
\end{tabular}

Table 7. Transformation from MN76 to present data

\begin{tabular}{lrr|lrr}
\hline & $x$-solution & & \multicolumn{3}{c}{$y$-solution } \\
\hline Term & coeff. & $\sigma$ & Term & coeff. & $\sigma$ \\
\hline offset & 0.30 & 0.20 & offset & 1.15 & 0.22 \\
$x(\mathrm{~cm})$ & -0.09 & 0.05 & $x(\mathrm{~cm})$ & -0.04 & 0.04 \\
$y(\mathrm{~cm})$ & -0.15 & 0.04 & $y(\mathrm{~cm})$ & -0.15 & 0.04 \\
$x * x$ & -0.085 & 0.013 & $x * y$ & -0.009 & 0.011 \\
$x * y$ & -0.115 & 0.012 & $y * y$ & -0.105 & 0.012 \\
mag-10 & -0.06 & 0.10 & mag-10 & -0.48 & 0.09 \\
$\mu_{x}$ & 0.055 & 0.006 & $\mu_{y}$ & 0.045 & 0.010 \\
$\mu_{y}$ & -0.002 & 0.011 & $\mu_{x}$ & 0.014 & 0.006 \\
\hline
\end{tabular}

\begin{tabular}{ll|ll} 
Reference stars : & 100 & unit weight st.dev.: & 1.46
\end{tabular} differences in proper motions between JW88 and the current study, before and after transformation. The scale of the figure is the same as for Figs. 5, 7 and 9. It was found that, in transforming the JW88 proper motions to the present study, we had to eliminate those stars for which errors of the proper motion were quoted to be larger than $0.8 \mathrm{mas} / \mathrm{yr}$, just as we did with our own data. After removing these data, a satisfactory transformation was obtained, as is shown in Fig. 6 and Table 8.

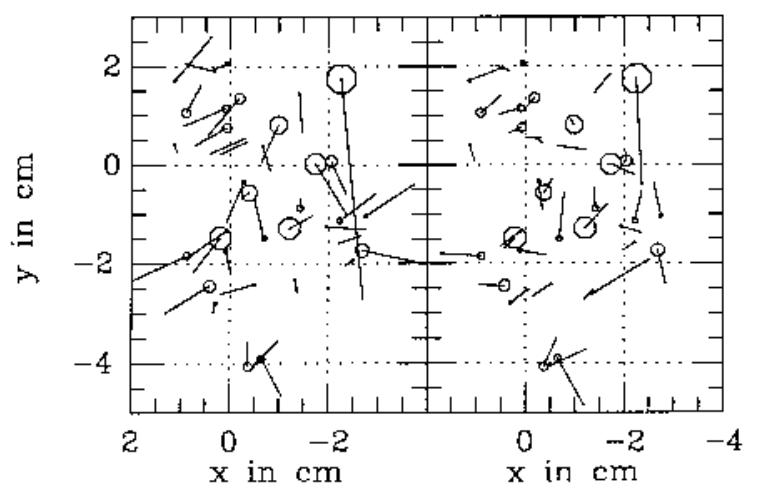

Fig. 8. Vectors on an $x-y$ chart of the field, representing the differences between proper motions presented by JW88 and the present paper; (left) before application of the transformation, and (right) after. Sizes of the circles refer to the magnitudes of the stars. A vector size corresponding to $1 \mathrm{~cm}$ in the position scale indicates a difference of $2.0 \mathrm{mas} / \mathrm{yr}$ 
Table 8. Transformation from JW88 to present data

\begin{tabular}{lrr|lrr}
\hline & $x$-solution & & \multicolumn{3}{c}{$y$-solution } \\
\hline Term & coeff. & $\sigma$ & Term & coeff. & $\sigma$ \\
\hline offset & -0.84 & 0.20 & offset & 0.61 & 0.20 \\
$x(\mathrm{~cm})$ & 0.17 & 0.23 & $x(\mathrm{~cm})$ & -0.32 & 0.16 \\
$y(\mathrm{~cm})$ & -0.01 & 0.09 & $y(\mathrm{~cm})$ & -0.07 & 0.12 \\
$x * x$ & 0.42 & 0.13 & $x * y$ & -0.53 & 0.11 \\
$x * y$ & 0.23 & 0.13 & $y * y$ & -0.02 & 0.05 \\
mag-12 & -0.02 & 0.12 & mag-12 & -0.05 & 0.10 \\
\hline \multicolumn{2}{l}{ Reference stars : } & 251 & unit weight st.dev.: & 1.34 \\
\hline
\end{tabular}

\subsection{The study by McNamara et al. (1989) (MN89)}

In this study 41 plates taken between 1911 and 1921, and 45 plates taken in 1987, all with the Harvard Metcalf telescope $\left(97^{\prime \prime} / \mathrm{mm}\right)$ were used. An area of some 2.6 by 2.6 was investigated and compared with the three studies mentioned above. Considerable problems were encountered in the comparison between MN89 and the present data. After transformation, the unit weight of standard error of the differences remained at a level of 1.8 , independent of whether only cluster members or all stars were used, whether a cutoff in magnitude was applied, or whether the field compared was reduced in size. There is an indication (in a graph not included here) from the differences in proper motion as vectors starting from the star-positions, that a main contributor to the problem is a distortion, either in the current study or in MN89, or in both. There appears to be a fourth order polynomial relation between residuals and coordinates, but too few points are available to make a sensible solution for it.

In the comparison it was noted that the proper motion for star Parenago 1876 as given by MN89 is wrong: the values $\left(\mu_{x}=-202.4 \pm 2.0 \mathrm{mas} / \mathrm{yr}, \mu_{y}=11.2 \pm 1.3 \mathrm{mas} / \mathrm{yr}\right)$ are not reproduced in any other paper. In our study, as well as in VA88 and Parenago (1954), this star has a very small proper motion and is a probable cluster member. Figure 6 and Table 9 summarize the results obtained with the transformation.

\subsection{Conclusions from comparisons}

The comparisons presented above show that we can rely upon the proper motions presented in the present paper when errors are below 0.8 mas/yr, although these errors may have been underestimated or the system may contain slight distortions. These uncertainties have to be taken into account when examining the proper motions for signs of internal motions. It was also shown that although differential proper motion studies can be very precise, with standard errors well below 1 mas/yr, the exact reference frame within which these proper motions are defined is not
Table 9. Transformation from MN89 to present data

\begin{tabular}{lrr|lrr}
\hline & $x$-solution & & \multicolumn{3}{r}{$y$-solution } \\
\hline Term & coeff. & $\sigma$ & Term & coeff. & $\sigma$ \\
\hline offset & -0.43 & 0.11 & offset & -0.23 & 0.13 \\
$x(\mathrm{~cm})$ & -0.034 & 0.018 & $x(\mathrm{~cm})$ & 0.031 & 0.020 \\
$y(\mathrm{~cm})$ & 0.103 & 0.014 & $y(\mathrm{~cm})$ & -0.014 & 0.017 \\
$x * x$ & 0.018 & 0.003 & $x * y$ & -0.007 & 0.004 \\
$x * y$ & 0.008 & 0.003 & $y * y$ & -0.014 & 0.003 \\
mag-12 & 0.17 & 0.04 & mag-12 & -0.18 & 0.05 \\
\hline \multicolumn{2}{l}{ Reference stars : } & 376 & unit weight st.dev.: & 1.83 \\
\hline
\end{tabular}

always easy to reproduce. Furthermore it appears (as must have been observed many times before) that when small numbers of plates are available, proper motion solutions can quite easily iterate towards a seemingly reasonable, but in fact, wrong solution, particularly when data are disturbed by varying background on different exposures.

A possibly somewhat disturbing feature in the transformations shown in Tables 6 to 9, is the size of the magnitude coefficient in the $y$-coordinate, which seems to indicate from the three studies that there is a magnitude dependance for the proper motions in the current study, even though any such relation present in the cluster stars in the current data had been removed (as can also be seen in Fig. 1). The problem could be related to the fact that many of the brightest stars in the field are close to or even embedded in nebulosity, which may have affected the proper motion determination in any of the studies mentioned, including the current one. There appears to be no such magnitude term present in the comparison between JW88 and the current study.

\section{The Orion cluster?}

The area under investigation in the present paper spans 13.1 by $14.8 \mathrm{pc}$ at the distance of Orion $(470 \mathrm{pc})$. This is a large area for an open cluster (e.g. the total diameter of the Pleiades is approximately 16 to $20 \mathrm{pc}$, with the central condensation occupying an area of about $3 \mathrm{pc}$ diameter). It is therefore surprising that, although we find a very strong concentration in the proper motions, there is no noticeable concentration in the projected positions of the stars. In fact, as was shown in Fig. 7 of JW88, the main feature of the "cluster" stars - a concentration along a band at constant RA - is a crescent-like feature extending over 10 degrees, which is more than $80 \mathrm{pc}$. The reduction methods remove the possibility of detecting linear rotation or expansion in the complex; these effects are lost in the necessary plate transformations.

The algorithms used to calculate membership probabilities also provide the standard deviation of the internal 


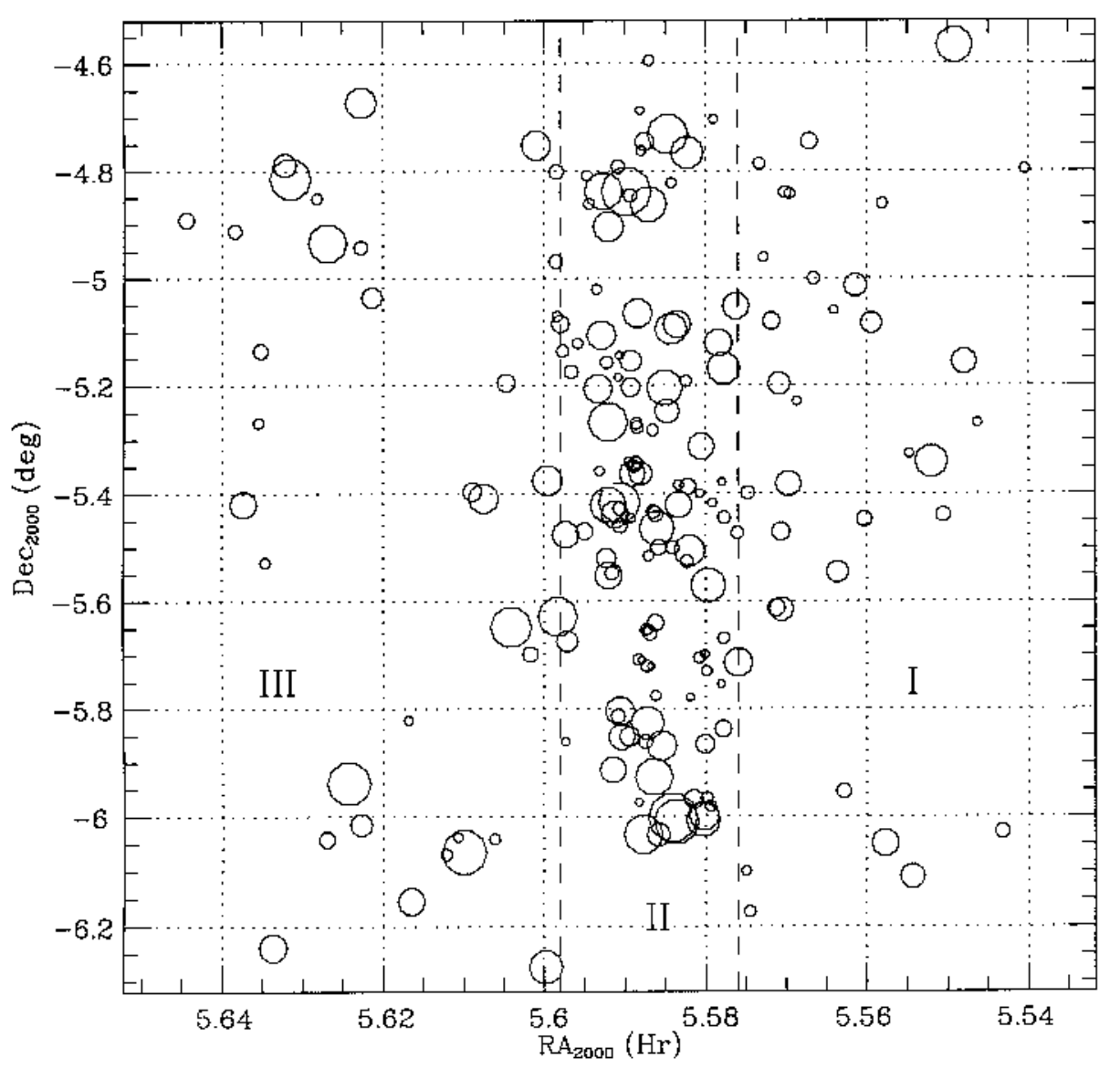

Fig. 9. The distribution of probable members in the Orion Nebula cluster field. The magnitudes are indicated by the sizes of the circles; the three parts of the field studied for proper motion dispersions are separated by dashed lines and indicated with Roman numerals

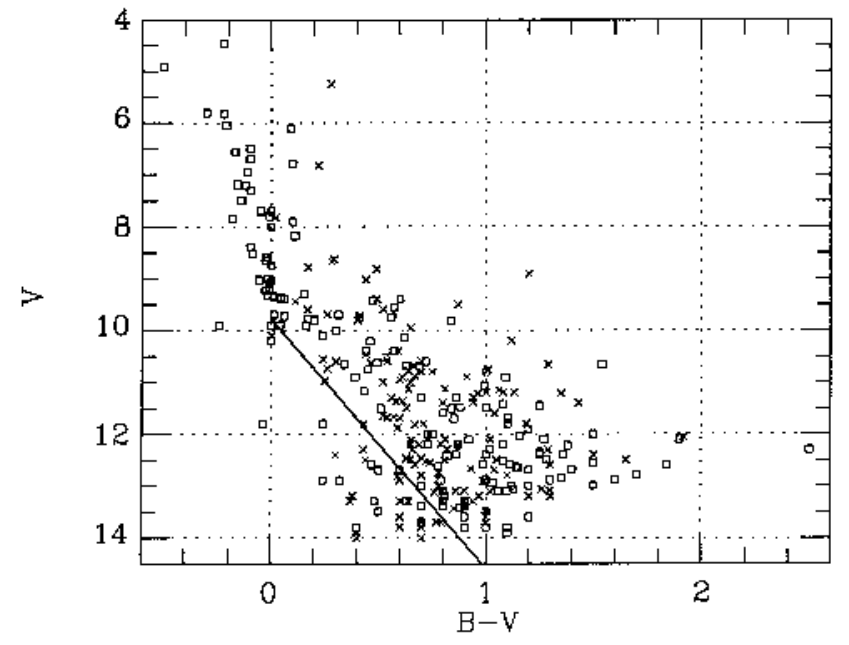

Fig. 10. The HR diagram for members (open squares) and non-members (crosses) of stars in the Orion Nebula cluster field (apparently filled symbols are due to superposition of an open square and a cross). The Pleiades locus shifted to a distance of $470 \mathrm{pc}$ is also shown
Table 10. Proper motion dispersions (in mas/yr) in different parts of the field. $N$ is the number of stars in each band

\begin{tabular}{lllllr}
\hline Band & Range in RA $\left(5^{\mathrm{h}}+\right)$ & $\sigma \mu_{x}$ & $\sigma \mu_{y}$ & $N$ & Width \\
\hline I & $32^{\mathrm{m}} 25^{\mathrm{s}}<R A<34^{\mathrm{m}} 34^{\mathrm{s}}$ & 0.97 & 1.24 & 28 & 0.54 \\
II & $34^{\mathrm{m}} 34^{\mathrm{s}} \leq R A \leq 35^{\mathrm{m}} 54^{\mathrm{s}}$ & 0.80 & 1.01 & 80 & 0.33 \\
III & $35^{\mathrm{m}} 54^{\mathrm{s}}<R A<38^{\mathrm{m}} 45^{\mathrm{s}}$ & 1.03 & 1.05 & 31 & 0.71 \\
\hline
\end{tabular}

proper motions of the cluster stars. Assuming the errors of the current proper motions to be correct, this gives the value presented in Table 4: 0.95 mas/yr, equivalent at a distance of $470 \mathrm{pc}$ to an upper limit for the internal velocity dispersion of $2.2 \pm 0.1 \mathrm{~km} / \mathrm{s}$. If we assume that our error estimates are too small by $35 \%$, this value decreases to $2.1 \pm 0.1 \mathrm{~km} / \mathrm{s}$. The change is very small, as can be expected when the errors of the proper motions are substantially smaller than the internal dispersion. The values found here are comparable to those found by JW88, and 
the discussion in that paper, considering the question as to whether the cluster is bound, still very much applies.

The distribution of the cluster member stars in the field, as shown in Fig. 9, does not justify an interpretation in terms of transverse and radial velocities which assumes an underlying spherically symmetric system. Instead, we looked at variations in the proper motion dispersions related to the main feature in the projected distribution of the stars. Three areas were chosen: the band containing the largest concentration of members as well as the nebulae (width 0.33) (II), the area to the east of this band (III), and the area to the west of it (I). Each band extends over the full declination range of the plate, 1:8. Table 10 summarizes the results obtained. There is an indication of a higher proper motion dispersion along the central area than perpendicular to it, but the differences are small.

Walker (1982) did an extensive spectroscopic study of stars in the same field as the current study. Comparing his membership classifications and criteria the following observations can be made. Firstly, there are five stars (Parenago 1350, 1519, 1732, 1743 and 2494) which both by McNamara et al. (1989) and the current study were assigned a $0 \%$ or low membership probability, but which do show characteristics similar to confirmed members. Secondly, there are three stars (Parenago 2068, 2303 and 2317) which were selected as members by Walker (1982) on the basis of their radial velocities, but which do not appear to be members according to the proper motions data of McNamara et al. (1989) or the current study. The first observation seems to suggest, like the proper motion dispersion and the distribution of stars on the sky, that the "Orion Cluster" is not a bound system: stars are quite freely moving away from the system from which they originated. The second observation shows once again the uncertainty in determining membership on the basis of one velocity component alone. On the other hand, given the very small proper motion of the cluster, the distinction between background stars and cluster members on the basis of proper motions alone is also quite uncertain, as can be seen in the HR diagram of the members and non-members shown in Fig. 10. This HR-diagram shows hardly any distinction between member and non-member stars. It appears from Fig. 10, as can be expected under these conditions, that among the faintest stars a number of non-cluster members on the far side of the cluster were assigned a high probability cluster membership.

\section{Conclusions}

The proper motions of stars in the region of the Orion Nebula confirm that stars in this region are still very close to the region where they were formed: proper motion dispersions are still very low. It may well be possible, that from this region at some stage, one or more star clusters will be formed, but presently the velocity dispersion and the distribution of the stars on the sky indicate that this has not (yet) happened for the majority of the stars observed here. An exception is probably the Trapezium cluster and its immediate surroundings (see also Herbig 1982), but they could not be included in the present study.

The study of differential proper motions turns out to be vulnerable to a variety of effects that are difficult or, indeed, almost impossible to detect from one study alone. These effects, which may include tilt-angle differences between old and new epoch plates and distortions due to changes in optics, may introduce some additional dispersion in the proper motions.

Acknowledgements. We would like to thank Rudolf S. Le Poole, F. van Rooyen and Cor de Vries for their assistance in measuring the plates on the ASTROSCAN at Leiden Observatory. We also like to thank Drs. L.V. Morrison and M.J. Penston who read the manuscript and acknowledge Mr. S. Chevalier who took the first epoch plates at Zô-Sè. This research was partly supported by the Royal Society and the Royal Dutch Academy of Sciences.

\section{References}

Bastian U., Röser S., 1993, PPM Star Catalogue, Vol. III, Spektrum Akad. Verl., Heidelberg

Chevalier S., 1905, Annales de l'observatoire de Zô-Sè 1, 1 Chevalier S., 1930, Annals of the Zô-Sè Observatory 18, 1

Herbig G.H., 1974, PASP 86, 604

Herbig G.H., 1982, Ann. NY Acad. Sci. 395, 64

$\mathrm{H} \varnothing \mathrm{g}$, et al., 1995, A\&A (in preparation)

Johnson H.M., 1965, ApJ 142, 964

Jones B.F., Walker M.F., 1988, AJ 95, 1755

Murray C.A., Nicholson W., 1975, in Image processing techniques in astronomy. In: de Jager C. and Nieuwenhuijzen H. (eds.). Reidel, Dordrecht, p. 171

McNamara B.J., 1976, AJ 81, 375

McNamara B.J., Hack W.J., Olson R.W., Mathieu R.D., 1989, AJ 97,1427

McNamara B.J., Huels S., 1983, A\&AS 54, 221

Parenago P.P., 1954, Trudy Sternberg Astron. Inst. Vol. 25

Sanders W.L., 1971, A\&A 14, 226

Strand K.A., 1958, ApJ 128, 14

van Altena W.F., Lee J.T., Lee J.-F., Lu P.K., Upgren A.R., 1988, AJ 95, 1744

van Leeuwen F., 1983, PhD Thesis, Leiden University

van Leeuwen F., 1994, in Galactic and Solar System Optical Astrometry. In: Morrison L.V. and Gilmore G.F. (eds.). Cambridge University Press, p. 223

Vasilevskis S., van Leeuwen F., Nicholson W, Murray C.A., 1979, A\&AS 37, 333

Vries C. de, 1986, PhD Thesis, Leiden University

Walker M.F., 1969, ApJ 155, 447

Walker M.F., 1982, ApJ 271, 642

Zhao J.L., He Y.P., 1990, A\&A 237, 54 